\title{
Propuesta De Mejoramiento De La Productividad De Las Pymes Transformadoras De Plástico Del Barrio Carvajal De Bogotá Mediante El Modelo De Productividad Gte.
}

\author{
Wilmar Suarez Gaitán dol , Marly Fernanda Calderón Pino, Aida Lorena Cedeño Buitrago \\ Universidad Autónoma De Colombia
}

Recibido: 15/04/2021 Revisado: 21/05/2021 Aceptado: 26/06/2021 Publicado: 28/07/2021

\section{Resumen}

La investigación "Propuesta de mejoramiento de la productividad de las pymes transformadoras de plástico del Barrio Carvajal de Bogotá mediante el modelo de productividad GTE”, busca por medio del modelo GTE incrementar la productividad teniendo en cuenta los factores tangibles e intangibles que afectan a las empresas del sector plásticos en la ciudad de Bogotá. Se pretende elaborar un diagnóstico de la situación actual de las empresas, diseñar una propuesta del modelo GTE y evaluar una propuesta de costos para su implementación. Para ello, el tipo de investigación es cuantitativo, disciplinar, aplicativo y descriptivo con el fin de identificar la situación actual en las empresas y diseñar e implementar el modelo de productividad (GTE) que traiga beneficios de productividad con soluciones a las problemáticas existentes. Los resultados esperados son: dar a conocer a los empresarios estrategias y mejoras frente a los factores internos y externos que los afectan, demostrar que el manejo eficiente de los recursos tiene una relación directa con la eficiencia, productividad y disminución en costos, demostrar que la motivación en las personas es un factor importante en el incremento de la productividad y brindarle al empresario un plan de mejoramiento que le traiga beneficios a las empresas.

\section{Palabras claves}

Productividad, modelo GTE, sector plástico, procesos productivos, recursos humanos. 


\begin{abstract}
The research "Proposal to improve the productivity of plastic transformer SMEs in the Carvajal neighborhood of Bogotá through the GTE productivity model", seeks through the GTE model to increase productivity taking into account the tangible and intangible factors that affect companies of the plastics sector in the city of Bogotá. It is intended to prepare a diagnosis of the current situation of the companies, design a proposal for the GTE model and evaluate a cost proposal for its implementation. For this, the type of research is quantitative, disciplinary, applicative and descriptive in order to identify the current situation in companies and design and implement the productivity model (GTE) that brings productivity benefits with solutions to existing problems. The expected results are: to make entrepreneurs aware of strategies and improvements in the face of internal and external factors that affect them, to demonstrate that the efficient management of resources has a direct relationship with efficiency, productivity and reduction in costs, to demonstrate that the Motivation in people is an important factor in increasing productivity and providing the employer with an improvement plan that brings benefits to companies.
\end{abstract}

\title{
Keywords
}

Productivity, GTE model, plastic sector, productive processes, human resources.

\section{INTRODUCCIÓN}

El problema de investigación es ¿Cómo se puede incrementar la productividad de las empresas pymes de plástico en Bogotá implementando el modelo GTE teniendo en cuenta la resolución 668 del 2016? Para ello, nuestro objetivo de investigación es diseñar una propuesta de mejoramiento de la productividad de pymes transformadoras de plástico del barrio Carvajal de Bogotá mediante el modelo de Gestión Tecnológica Empresarial (GTE) de la productividad, teniendo en cuenta la implementación de la resolución 668 de 2016 sobre el plástico. Entre los objetivos que se pretenden alcanzar están el objetivo específico que es: Elaborar un diagnóstico de la situación actual de las empresas seleccionadas para la investigación mediante el modelo de gestión tecnológica empresarial (GTE) de la productividad, diseñar una propuesta de mejora y de costos para las empresas seleccionadas para la investigación determinando la situación presente en cada una de las áreas de las empresas a investigar por medio del uso de herramientas de ingeniería industrial y analizando los factores tangibles e intangibles que se ajusten al modelo a trabajar.

\section{MARCO TEÓRICO}


Productividad: En Prokopenko (1989), el autor afirma que la productividad: "La productividad da buenos resultados a las empresas en relación a la eficacia y cumplimiento de objetivos y el proceso de producción en una empresa debe tener una integridad entre entorno, trabajo y capital para cumplir con el buen funcionamiento y el mejoramiento de la productividad. Entre los factores de productividad propuesta por Mukherjee y Singh están los internos o controlables y externos o no controlables". (Prokopenko, 1989, pp. 3-9)

La medición de la productividad total: Para medir la productividad total en las empresas, Sumanth afirma que: "Se debe tener en cuenta los productos de fábrica, midiendo las productividades parciales en términos reales teniendo en cuenta un periodo base dividiendo los valores de la producción y los insumos o recursos utilizados”. (Sumanth, 1990, p. 547)

Productividad parcial: La productividad parcial establece: "la conexión entre los productos elaborados en una empresa y cada uno de los recursos o insumos utilizados para la elaboración de los mismos entre ellos: tiempo, mano de obra, materiales, etc. La fórmula es: Productividad parcial= salida total/ una entrada”. (Carro \& González, 2012, p. 5)

Productividad total: Según Carro \& González (2012), la productividad total es: "La relación entre la salida referente a lo producido en la fábrica en relación al conjunto total de las entradas ósea todos los recursos necesarios para la elaboración del producto final. Productividad total $=$ bienes y servicios producidos/mano de obra + capital + materias primas + otros”. (Carro \& González, 2012, p. 5)

Factores que restringen la productividad: "Los factores que no ayudan a que las empresas sean productivas, son a causa de no manejar un ambiente y clima propicio para el mejoramiento de la misma, no cuentan con reglamentos gubernamentales, les falta una estructura adecuada donde el diseño y redistribución de planta sean fundamental para cumplir los objetivos productivos, la falta de medición, capacidad de las empresas, abastecimiento, diseño de nuevos productos, maquinaria, equipo y la calidad en la materia prima generan perjuicios pues no tienen un control de cada aspecto de la organización”. (García, 2005, pp. 1011)

Sector de plásticos: "El plástico es uno de los materiales más comunes y más usados en el mundo, a nivel industrial todo plástico pasa por un proceso de transformación que lo vuelve más resistente o más ligero dependiendo de la necesidad del cliente”. (Morillas, Villavicencio. Espinoza y Pérez, 2018, p. 4) 
"Las empresas dedicadas al sector de plásticos en Colombia son muy importantes ya que aportan a la economía del país el 15\% del PIB y es significativo en temas de generación de empleos con cerca de 65.000 empresas y aportación a otros sectores importantes en Colombia con 2.500 establecimientos dedicados al plástico y similares”. (Dinero, 2018) Según las estadísticas del DANE en el Boletín técnico de encuesta anual manufacturera (EAM) 2018, el sector dedicado a los plásticos ocupa el séptimo lugar en aportación al valor agregado con un porcentaje de 4,3\% del total de industrias aportantes del país, con una contribución de producción bruta del $4.1 \%$ en el sexto puesto con $\$ 10.746 .867$ y una generación de empleo del 7,8\% con 55.472 en 2018. (DANE, 2018)

Modelo de productividad GTE - gestión tecnológica empresarial: El modelo GTE fue creado por el grupo de investigación de la facultad de ingeniería industrial de la Universidad Autónoma de Colombia de acuerdo a la revisión de diferentes modelos y teorías acerca de la productividad con el fin de contar con una herramienta que permita ser aplicada en las empresas del sector plástico y dar soluciones a las problemáticas que se presentan en relación a las áreas administrativas, productivas, recurso humano y logística en dónde se determinan aquellas variables tangibles e intangibles que puedan ser evaluados y buscar estrategias que incrementen beneficios a las empresas sabiendo que las empresas.

Resolución 668 del 2016: En el 2016, el gobierno nacional implementó la resolución 668 del 2016 la cual tiene por objeto: "que los distribuidores de bolsas plásticas deben formular, presentar, implementar y estar registrados en el uso racional de los productos que venden. En las bolsas debe estar visible mensajes ambientales, la capacidad en kilogramos y el calibre, en los almacenes las bolsas deben cumplir con un tamaño estándar de $30 \mathrm{~cm}$ x 30 $\mathrm{cm}$, un calibre de 0.9 milésimas de pulgada y contar con una alternativa con ventas de bolsas reutilizables”. (Ministerio de Ambiente y Desarrollo Sostenible - República de Colombia, 2016).

\section{METODOLOGÍA}

Para cumplir con la investigación se recolectó información primaria para determinar el diagnóstico actual de las empresas a investigar e información secundaria con información sobre el sector de plásticos, teorías de la productividad y estadísticas del sector plástico. Luego se determinó las variables que afectan la productividad en las empresas estudiadas, se realizó matrices de valoración de importancia de las variables seleccionadas, se diseñó el modelo de productividad GTE que sirve para aplicarlo en las empresas, se implementó el modelo pudiendo obtener 
resultados favorables y demostrar la eficacia del modelo que busca incrementar las ventas y la eficacia en la empresa con planes de mejora para los empresarios. El universo es el diagnóstico, el diseño y la implementación del modelo GTE en las empresas del sector plástico en Bogotá y la población y la muestra son las 10 empresas del sector pymes en Bogotá en las áreas administrativas, productivas, recurso humano y logística. El tipo de investigación que usaremos será cuantitativo, disciplinar, aplicativo y descriptivo realizando un estudio de las diferentes empresas con el fin de identificar la situación actual y dar soluciones para incrementar la productividad.

\section{DESARROLLO DEL TRABAJO}

\subsection{Proceso diagnóstico:}

En Mayorga y Porras (2015), los investigadores de la Universidad Autónoma de Colombia desarrollan una revisión del estado de la productividad de las Pymes del sector del caucho y el plástico en Bogotá al año 2015. En la publicación se trata la falta de competitividad derivada

principalmente del bajo nivel productivo de la industria del sector, los problemas de distribución y comercialización y el deficiente direccionamiento estratégico de las empresas; por lo que el estudio se orientó a analizar el entorno interno y externo del sector, su desempeño productivo y su proyección en el mercado internacional. (Mayorga \& Porras, 2015)

\subsection{Recopilación de la información:}

Se realizó un análisis detallado de una muestra de empresas de la capital colombiana, evaluando sus áreas hasta llegar a una modelación econométrica que mide la productividad de las industrias del sector del plástico y el caucho, con base en la metodología del valor agregado y su regresión lineal, incluyendo su validación a través de pruebas de validación estadística.

\subsection{Procesamiento de la información:}

Posteriormente, se presentan los resultados de la aplicación del modelo, comenzando con el análisis básico del desempeño del sector con base en las estadísticas publicadas por el DANE a 2012, pasando por la revisión de la productividad laboral, del costo laboral y de los niveles salariales, llegando a la caracterización de las empresas del sector a través de la aplicación de encuestas a la muestra de estudio. Esa caracterización tuvo en cuenta la gestión administrativa y talento humano, procesos, mercadeo y financiera. Se pasó, luego, a la aplicación del modelo econométrico en sí mismo y analizando los indicadores de la metodología del valor agregado. 


\subsection{Análisis de la información:}

De acuerdo a la encuesta realizada como método de recolección de información pudimos evidenciar la situación actual de 10 empresas pymes de plástico teniendo en cuenta el diseño de la matriz de priorización como se observa a continuación:

\begin{tabular}{cl}
\hline Puntuación & \multicolumn{1}{c}{ Descripción nivel } \\
\hline $\mathbf{1}$ & $\begin{array}{l}\text { Nivel leve de impacto frente a la } \\
\text { productividad no afecta a la empresa en } \\
\text { la competencia. }\end{array}$ \\
\hline $\mathbf{2}$ & $\begin{array}{l}\text { Nivel medio de impacto frente a la } \\
\text { productividad hace que la competencia } \\
\text { de la empresa sea inestable }\end{array}$ \\
\hline $\mathbf{3}$ & $\begin{array}{l}\text { Nivel alto de impacto frente a la } \\
\text { productividad hace que la empresa esté } \\
\text { en riesgo de salir de competencia }\end{array}$ \\
\hline
\end{tabular}

Tabla 1 Puntuación de impacto frente a la productividad de las empresas

\subsubsection{Resultados área administrativa:}

En el área administrativa, el 100\% de las empresas encuestadas cuentan con normas legales de funcionamiento, se encuentran registradas en cámara de comercio y tienen definidos sus objetivos organizacionales. Adicional a esto, las empresas cuentan con portafolio de productos definidos más sin embargo el 30\% de las empresas encuestadas no manejan estrategias de publicidad como páginas web. Además, el $60 \%$ de las empresas no cuentan con software que les permite tener un control de los recursos utilizados en cada una de las áreas de la empresa lo que incurre a costos innecesarios. El 90\% de las empresas están en contacto con el cliente en temas de devoluciones, servicios postventa y atención PQRS. 


\begin{tabular}{|c|c|c|c|c|c|c|}
\hline \multirow{3}{*}{$\begin{array}{l}.^{\circ} \text { DE } \\
\text { EMPRESA } \\
\text { S } \\
\end{array}$} & \multirow[b]{3}{*}{ N. ${ }^{\circ}$} & \multirow{3}{*}{$\begin{array}{l}\text { AREA ADMINISTRATIVA } \\
\text { Observaciones }\end{array}$} & \multirow{3}{*}{$\begin{array}{l}\text { ENCUESTAS } \\
\text { PORCENTAJE } \\
\text { S }\end{array}$} & \multicolumn{3}{|c|}{ Priorización } \\
\hline & & & & & & \\
\hline & & & & 1 & 2 & 3 \\
\hline \multirow[t]{6}{*}{10} & 1 & $\begin{array}{l}\text { Las empresas cuentan con las } \\
\text { normas legales de funcionamiento }\end{array}$ & $100 \%$ & & & $\mathrm{X}$ \\
\hline & 2 & $\begin{array}{l}\text { Las empresas cuentan con } \\
\text { portafolio de productos }\end{array}$ & $100 \%$ & & & $\mathrm{X}$ \\
\hline & 3 & $\begin{array}{l}\text { No manejan estrategia de } \\
\text { publicidad }\end{array}$ & $30 \%$ & & $\mathrm{X}$ & \\
\hline & 4 & $\begin{array}{l}\text { No cuentan con un software } \\
\text { especializado para el control de los } \\
\text { recursos utilizados en cada área }\end{array}$ & $60 \%$ & & $\mathrm{X}$ & \\
\hline & 5 & $\begin{array}{l}\text { Mantienen contacto con el cliente } \\
\text { en temas de devoluciones, servicios } \\
\text { postventa y atención } \mathrm{PQRS} \text {. }\end{array}$ & $90 \%$ & & $\mathrm{X}$ & \\
\hline & & $\begin{array}{l}\text { Ponderación por área }=\text { Total } \\
\text { Puntos } \mathrm{x}(0,25 / \text { No de observaciones } \\
\text { del área }) \text {. }\end{array}$ & $\begin{array}{l}\text { Puntaje Ponder } \\
12 *(0,25 / 5)=0\end{array}$ & & & \\
\hline
\end{tabular}

Tabla 2. Tabla de resultados área administrativa

\subsubsection{Resultados área productiva:}

En el área productiva, el $100 \%$ de las empresas encuestadas cuentan con sus procesos definidos para la elaboración de plásticos, aunque no tienen documentados los procesos. En cuanto a la distribución en planta, el $60 \%$ de las empresas cuentan con el espacio adecuado para el proceso de fabricación de sus productos, aunque se requiere de estudio de tiempos y movimientos para optimizar tiempos en transporte, y eliminación de actividades que se pueden realizar en el mismo proceso. Además, el $80 \%$ de las empresas realizan programas de mantenimiento preventivo, calibración de herramientas y cuentan con herramientas de seguridad en caso de averías y en un 90 \% cuentan con sistema de verificación e inspección de los productos fabricados.

\begin{tabular}{|c|c|c|c|c|c|}
\hline $\mathrm{N}^{\circ}$ & & AREA PRODUCCION & ENCUESTAS & Prioriz & ción \\
\hline EMPRESAS & $\mathrm{N} .^{\circ}$ & Observaciones & PORCENTAJES & 1 & 3 \\
\hline 10 & 1 & Buena distribución de planta & $60 \%$ & & $\mathrm{X}$ \\
\hline & 2 & $\begin{array}{l}\text { Se cuenta con el mantenimiento } \\
\text { preventivo }\end{array}$ & $80 \%$ & $\mathrm{X}$ & \\
\hline & 3 & $\begin{array}{l}\text { Cuentan con sistema de verificación e } \\
\text { inspección de los productos } \\
\text { elaborados en temas de calibración, } \\
\text { capacidad y resistencia }\end{array}$ & $90 \%$ & & $\mathrm{X}$ \\
\hline & & $\begin{array}{l}\text { Ponderación por área }=\text { Total Puntos } \\
x(0,25 / \text { No de observaciones del } \\
\text { área). }\end{array}$ & $\begin{array}{l}\text { Puntaje Ponderad } \\
8^{*}(0,25 / 3)=0,66\end{array}$ & $\begin{array}{l}\text { total } 8 \\
\text { lo }=\end{array}$ & \\
\hline
\end{tabular}

Tabla 3 Tabla de resultados área productiva 


\subsubsection{Resultados área de recursos humanos:}

En el área de recursos humanos, el 80 \% de las empresas no tienen documentación sobre los puestos de trabajo, de la correcta utilización de las herramientas y no cuentan con área de talento humano ya que todo lo manejan en el área administrativa. Además, las empresas en un $50 \%$ cuentan con incentivos por realizar bien su labor, y cuentan con capacitaciones y oportunidades de superación profesional lo cual se debe reforzar. En las empresas, el $100 \%$ realizan pausas activas puesto que el área productiva cuenta con posturas inadecuadas, repetitivas y con manejo de cargas de material y todas las empresas tienen aseguradora y los tienen afiliados a la EPS.

\begin{tabular}{|c|c|c|c|c|c|c|}
\hline \multirow{2}{*}{$\begin{array}{l}\text { N. }{ }^{\circ} \mathrm{DE} \\
\text { EMPRESAS }\end{array}$} & \multirow[b]{2}{*}{ N. ${ }^{\circ}$} & \multirow{2}{*}{$\begin{array}{l}\text { AREA RECURSO HUMANO } \\
\text { Observaciones }\end{array}$} & \multirow{2}{*}{$\begin{array}{l}\text { ENCUESTAS } \\
\text { PORCENTAJES }\end{array}$} & \multicolumn{3}{|c|}{ Priorización } \\
\hline & & & & 1 & 2 & 3 \\
\hline \multirow[t]{5}{*}{10} & 1 & $\begin{array}{l}\text { Las empresas no cuentan con área de } \\
\text { talento humano ya que todo lo } \\
\text { manejan en el área administrativa }\end{array}$ & $80 \%$ & & & $\mathrm{X}$ \\
\hline & 2 & $\begin{array}{l}\text { Las empresas cuentan con incentivos } \\
\text { capacitaciones y oportunidades de } \\
\text { superación profesional para sus } \\
\text { trabajadores }\end{array}$ & $50 \%$ & & & $\mathrm{X}$ \\
\hline & 3 & $\begin{array}{l}\text { Si se realizan pausas activas porque } \\
\text { en el área productiva se adoptan } \\
\text { posturas inadecuadas, repetitivas y } \\
\text { con manejo de cargas. }\end{array}$ & $100 \%$ & & & $\mathrm{X}$ \\
\hline & & & & \multicolumn{3}{|c|}{ total 9} \\
\hline & & $\begin{array}{l}\text { Ponderación por área }=\text { Total Puntos } x \\
(0,25 / \text { No de observaciones del área }) .\end{array}$ & $\begin{array}{l}\text { Puntaje Ponderad } \\
9 *(0,25 / 3)=0,75\end{array}$ & & & \\
\hline
\end{tabular}

Tabla 4 Tabla de resultados área de recursos humanos

\subsubsection{Resultados área de logística:}

En el área de logística, el $90 \%$ de las empresas seleccionan a los proveedores en función calidad-tiempo, así como también inspeccionan la materia prima y tienen en cuenta la puntualidad y seriedad en él envió. En un 80 \% las empresas no cuentan con indicadores que determinen los tiempos de demora de orden de pedido. Además, el $30 \%$ no cuentan con transporte de materia prima y productos terminados lo cual hace que el proveedor o cliente deba transportar su pedido y el $100 \%$ de las empresas dicen cumplir con las entregas de los pedidos puesto que son responsables y consientes en la capacidad que poseen. 


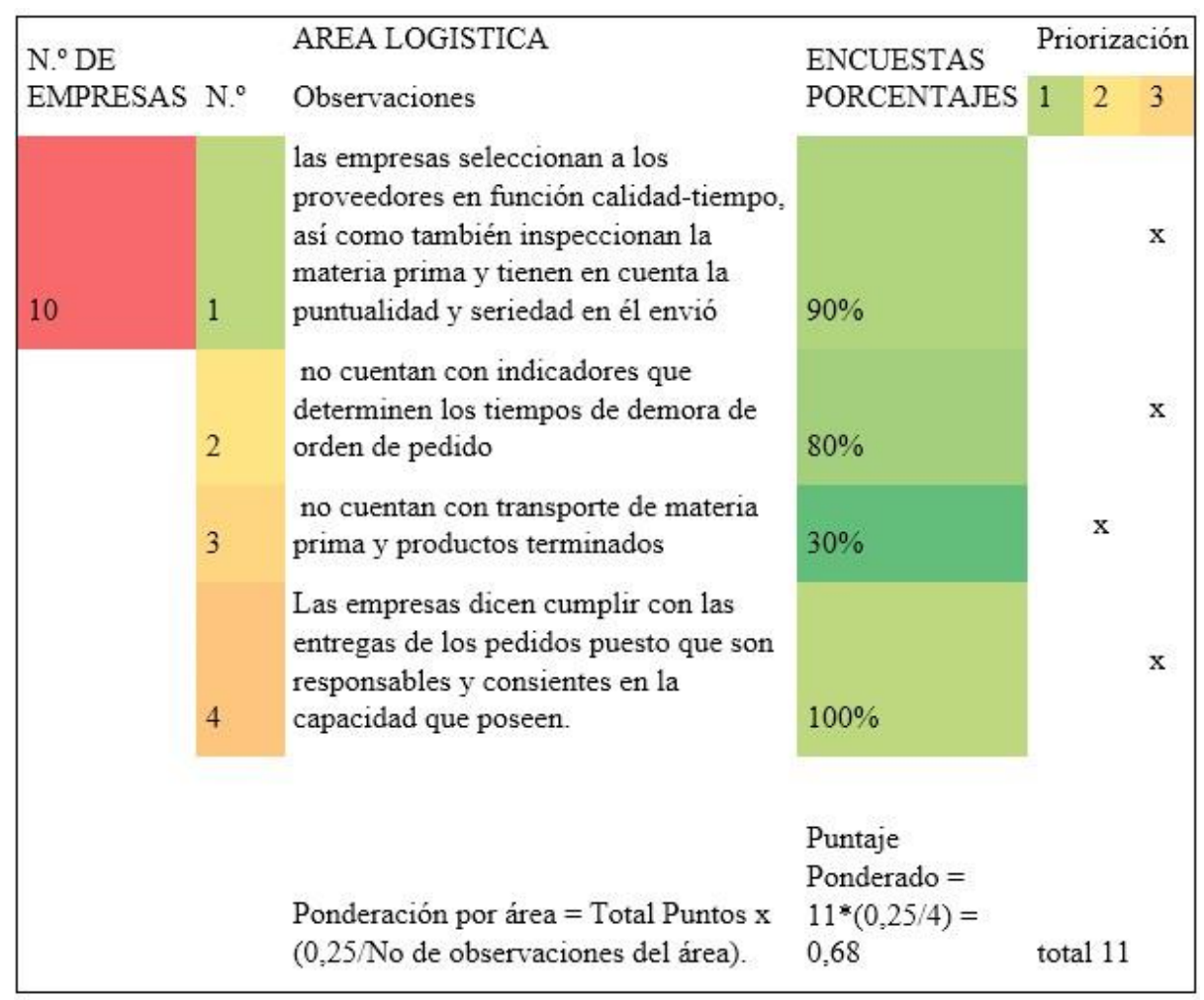

Tabla 5 Tabla de resultados área logística

\subsubsection{Resultados de la empresa:}

Después de realizar la matriz de priorización por cada una de las áreas podemos clasificar por nivel de importancia teniendo como resultado el área que genera mayor impacto en la productividad de las empresas.

\begin{tabular}{lc}
\hline ÁREAS DE LA & NIVEL DE \\
EMPRESA & $\begin{array}{l}\text { IMPORTANCIA } \\
\text { DENTRO DE LA } \\
\text { EMPRESA }\end{array}$ \\
\hline Recurso Humano & 1 \\
\hline Logística & 2 \\
\hline Producción & 3 \\
\hline Administración & 4 \\
\hline
\end{tabular}

Tabla 6 Priorización por área

\subsubsection{Determinación de la matriz de valoración de importancia de las áreas funcionales}

Se utilizó la herramienta ITU con la cual se establece el nivel de importancia en cada una de las áreas. A continuación, se presenta la convención de los puntajes por variable en el que se puede distinguir baja, media o alta afectación. 


\begin{tabular}{lc}
\hline \multicolumn{2}{c}{ Puntaje de las variables } \\
\hline Baja & 1 \\
\hline Media & 3 \\
\hline Alta & 5 \\
\hline
\end{tabular}

Tabla 7 Convención de los puntajes por variable

El área de producción es la que tiene mayor valoración, seguida del área de logística, el área administrativa y por último el área de recursos humanos de acuerdo a la incidencia de cada una de las áreas en las diferentes variables que afectan la productividad en las empresas como se observa en la tabla 8 .

\subsection{Socialización de los resultados:}

\begin{tabular}{|c|c|c|c|c|c|c|c|c|c|c|c|c|c|}
\hline $\begin{array}{c}\text { Área que } \\
\text { impacta la } \\
\text { productividad } \\
\text { en el empresa }\end{array}$ & \begin{tabular}{|c|} 
Nivel de \\
importanci \\
a dentro de \\
la empresa
\end{tabular} & $\begin{array}{l}\text { Participació } \\
\text { n en el } \\
\text { proceso }\end{array}$ & N. & $\begin{array}{c}\text { Participación } \\
\text { en el } \\
\text { procedimient }\end{array}$ & $\mathrm{N}$ & $\begin{array}{c}\text { Participació } \\
\text { n en los } \\
\text { proveedore }\end{array}$ & $\mathrm{N}$. & $\begin{array}{l}\text { Satisfacció } \\
\text { n del } \\
\text { cliente }\end{array}$ & N. & $\begin{array}{c}\text { Participa } \\
\text { c ión de } \\
\text { los } \\
\text { recursos }\end{array}$ & N. & TOTAL & $\begin{array}{l}\text { FRECUENCI } \\
\text { A } \\
\text { RELATIVA }\end{array}$ \\
\hline Recurso & 1 & 5 & 5 & 1 & 5 & 1 & 1 & 3 & 3 & 5 & 3 & 15 & $18,75 \%$ \\
\hline Logística & 2 & 5 & 10 & 5 & 10 & 5 & 10 & 3 & 6 & 3 & 6 & 21 & $26,25 \%$ \\
\hline Producción & 3 & 5 & 15 & 5 & 15 & 5 & 15 & 5 & 15 & 5 & 15 & 25 & $31,25 \%$ \\
\hline Administración & 4 & 5 & 20 & 3 & 12 & 3 & 12 & 5 & 20 & 3 & 20 & 19 & $23,75 \%$ \\
\hline Importancia en & & 50 & & 42 & & 38 & & 44 & & 44 & & 80 & $100,00 \%$ \\
\hline
\end{tabular}

Tabla 8 Identificación de prioridades en relación a las acciones a tomar

Las Pymes de esta industria no han podido hacer uso eficiente de recursos como maquinaria y tecnología por limitaciones como la disponibilidad de capital de inversión, la gestión administrativa, la falta de innovación y análisis de optimización y uso eficiente de los mismos.

\section{CONCLUSIONES}

En el desarrollo de este proyecto de investigación, se logró utilizar el modelo GTE como herramienta de medición de la productividad en las empresas generando recomendaciones para cada una de las pymes del sector de este sector realizando un análisis de priorización por cada área y teniendo en cuenta cada uno de los resultados obtenidos se realiza una planeación, control y mejora continua. Además, se demuestra la importancia de la motivación en el manejo de recursos humanos ya que incrementa la productividad personal, satisfacción de las necesidades personales y profesionales, mejoramiento del clima laboral y el correcto lineamiento de todos los miembros de la empresa encaminado a cumplir los objetivos de las empresas investigadas.

La mayoría de las empresas pymes de plástico en Bogotá tienen falencias en el área administrativa por la falta del seguimiento interno, constante y documentado en el que la alta dirección conozca la información de lo que sucede cada periodo en cuanto a las ventas y la producción, por consiguiente, no hacen un seguimiento, control y mejora continua para satisfacer 
a sus clientes por la falta de documentación, uso de software y estrategias en pro de aumentar la productividad y las ventas.

Se observó que el sector plástico en cuanto al manejo de producción y administración se da de forma empírica lo que conlleva a un bajo control de la producción, costos y finanzas principalmente. Por eso la importancia de la implementación de herramientas y de un modelo que generen solución a los problemas anteriores y contar con la rápida adecuación ante los cambios del entorno, con el fin de mejorar la situación actual y así mismo, mejorar sus finanzas, disminuyendo costos y utilizando de manera eficiente todos los recursos.

A pesar que el sector es competitivo la productividad es baja debido a las normativas ambientales y todos los factores externos que afectan a las empresas puesto que el plástico genera un gran impacto ambiental y no se cuenta con políticas del correcto manejo de los residuos sólidos que en la mayoría de los casos es de gran dispersión por el incremento del uso de este material y el proceso tan demorado de degradación el cual afecta al medio ambiente. Por estas razones es importante buscar estrategias e implementar el modelo de productividad GTE, mirando que soluciones se le pueden plantear a las empresas que están en proceso de investigación. 


\section{REFERENCIAS}

Carro, R. y González, D. (2012). Productividad y competitividad [Archivo PDF]. Recuperado de: http://nulan.mdp.edu.ar/1607/1/02_productividad_competitividad.pdf

DANE. (2018). Boletín Técnico. Encuesta Anual Manufacturera EAM 2018. [PDF en línea]. Recuperado de https://www.dane.gov.co/files/investigaciones /boletines/eam/boletin_eam_2018.pdf

Dinero. (13 de agosto de 2018). Las reformas que pide el sector plástico al presidente Duque. Dinero. Recuperado de: https://www.dinero.com/empresas/articulo/sector-plastico-encolombia-pide-reformas-a-ivan-duque/260890

García, R. (2005). Estudio del trabajo, ingeniería de métodos y medición del trabajo. McGraw Hill. Mayorga, J. \& Porras, J. (2015). Productividad de las Pymes, sector caucho y plástico de Bogotá D.C. [PDF en línea]. Recuperado de https://dialnet.unirioja.es/descarga/articulo/6634697.pdf

Morillas, A., Villavicencio, M., Espinoza, R y Pérez, M. El origen de los plásticos y su impacto en el ambiente [Archivo PDF]. Recuperado de: https://anipac.com/wpcontent/uploads/2018/09/origendelosplasticos.pdf

Prokopenko, J. (1989) La gestión de la productividad manual práctico. Ginebra.

Resolución 0668 de 2016. [Ministerio de Ambiente y Desarrollo Sostenible - República de Colombia]. Por la cual se reglamenta el uso racional de bolsas plásticas y se adoptan otras disposiciones. 28 de abril de 2016.

Sumanth, D. (1990). Ingeniería y Administración de la Productividad. 